\title{
Forecasting the Production Abilities of Recycling Systems: A DEA Based Research
}

\author{
Feng Yang, Fei Du, Liang Liang, and Zheng Yang \\ School of Management, University of Science and Technology of China, Hefei, Anhui 230026, China \\ Correspondence should be addressed to Zheng Yang; yang.som.ustc@gmail.com
}

Received 15 April 2014; Accepted 6 June 2014; Published 18 June 2014

Academic Editor: Xiaohang Yue

Copyright (C) 2014 Feng Yang et al. This is an open access article distributed under the Creative Commons Attribution License, which permits unrestricted use, distribution, and reproduction in any medium, provided the original work is properly cited.

\begin{abstract}
Network production systems are widely observed in the current real life, and the performance management of network production systems has been widely studied. The previous literatures have contributed to the performance evaluation to parallel, serial, and converging systems. However, few literatures have considered the recycling systems. The current paper proposes a method on how to forecast the actual and frontier production abilities of recycling systems. Based on the forecasting results, the paper uses a DEA (data envelopment analysis) based method to appraise the operational efficiency of recycling systems. We choose the water utilization and recycling system as a background for our research. According to the proposed method, Chinese provincial water utilization and recycling systems are examined and analyzed. The results show that the reuse level of water in China is still very low.
\end{abstract}

\section{Introduction}

The famous saying "no water, no life" is known by almost everyone. Maybewater is one of the most precious scarce resources on this rotating globe, especially in China, whose per capita amount of water resource is equivalent to about one-fourth of the world's average level. Over the past 30 years, China has seen the great development in the economy and society, while it has also suffered from the scarcity of resources, including energy and materials as well as water, especially in its western provinces where the scarcity of water has brought huge difficulties to family lives and economic development. Water utilization efficiency has received increasing attention from both policy makers and academic researchers, such as Zhong [1] and Varis and Vakkilainen [2].

Many studies have been made on the performance measure for water utilization. For example, Thanassoulis [3] employed data envelopment analysis (DEA) method, which was proposed by Charnes et al. [4], in the regulation of UK water utilities, and the related results were useful to rearrange the water distribution to obtain higher utilization potential. Jin and Young [5] described the situations of water distributions in China and discussed the impact of water distribution on Chinese agriculture. Their research showed that the imbalance of water distribution was one of the most important reasons why western China's economy was in a very low level while the eastern part was quite the opposite. Varis and Vakkilainen [2] insisted on the fact that a better utilization of water resource was the way to deal with the potential challenges of water scarcity in China. Zhong [1] gave some advice on water resources planning and management for sustainable development for China. Mo et al. [6] and Huang et al. [7] examined the water use efficiency and its impact on agriculture in North China. Hu et al. [8] extended their research to all industries and compared the regional water utilization efficiencies in China. Although the researches mentioned above have done a lot on water utilization efficiency, they did not consider the scenario that waste water was regenerated and reused. Considering the reuse of water would be helpful for better utilization of this resource. Nasiri and Huang [9] mentioned the repeated use of water; however, it is a pity that they did not calculate the whole circulation of how water is used, purified, and regenerated.

In fact, the whole process for water's utilization and reuse is an infinitely dynamic and recycling system. Färe and Grosskoft [10] and Lewis and Sexton [11] have proposed reasonable methods for performance assessment for network systems. The water utilization and recycling system is also a network system, but it is different from those examined 
by Färe and Grosskoft [10] and Lewis and Sexton [11], since circulation exists in the former. Thus more precise methods are needed to appraise the performance of dynamic and recycling systems.

In the above part we have discussed the water utilization efficiency in economic development. In practice, water utilization is also an important issue in environmental performance assessment. During the water utilization, some useful outputs are produced, while the waste water is also generated. The efficiency measure accounting for undesirable outputs becomes the foremost and popular technique in environmental performance assessment. Färe et al. [12, 13] have pioneered the existence of undesirable outputs and established the foundational appraising models for systems with undesirable outputs. Other articles in this line include Amirteimoori et al. [14], Hua et al. [15], and Liang et al. [16]. In the current paper, undesirable outputs are also involved in the performance assessment, and the existing methods are used for reference.

In the current paper, we focus on Chinese provincial water utilization and recycling system and point out that the system is a typical infinitely dynamic and recycling system. We try to propose a reasonable method to appraise the performance of that type of dynamic systems. To do that, we need to forecast the actual production abilities and frontier production abilities based upon the existing technique constraints for all of dynamic systems in the first step. After that, we compute the system performance by the ratio of the actual production ability to the frontier production ability. We also examine the Chinese provincial water utilization and recycling systems according to the results of our methods.

The rest of the paper is organized as follows: Section 2 describes Chinese provincial water utilization an recycling systems, including variables and subsystems. The foundational assumptions are given in Section 3. In Section 4, we advance the efficiency evaluation model for water utilization and recycling systems. Results analysis and further discussion are shown in Section 5. Finally conclusions and remarks are given in the last section.

\section{Descriptions of Water Recycling and Utilization System}

2.1. Water Recycling and Utilization System. In the industrial production, water is indispensable for almost all of the existing industries, such as iron and steel, paper mill, pharmacy, and tourism industry. Therefore water availability is often regarded as an important criterion in the facility location decision making.

For a microenterprise, its water utilization process is extremely simple. A microenterprise pays some monetary capital to waterworks and acquires the equivalent value of fresh water. In the production the water is consumed. As a result the water does usually not disappear but becomes waste water. At the end, the waste water is emitted. In general, a microenterprise does not treat water as a scarce resource and would usually not undertake the task of water regeneration and recycling.

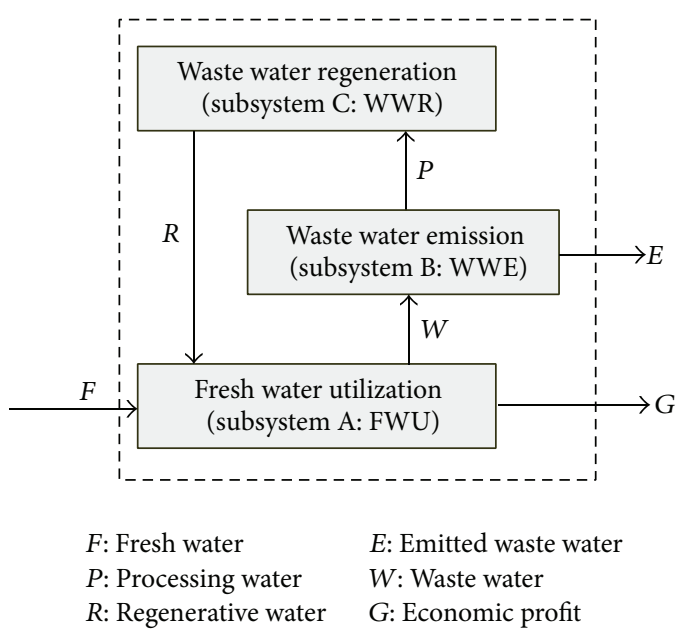

FIGURE 1: Water recycling and utilization.

From the perspective of a macroregion, city, or country, the situations are totally different. We only talk about industrial water here. For example, a city should consume plenty of water every day. There are few cities which have exhaustless fresh water if no regenerated water is produced there. Accordingly, water is usually a scarce resource for a city, as well as a macroregion or a country. During the normal operation of a city, fresh water is consumed by the industries; part of the waste water is transferred back to the waterworks and the other is emitted directly; finally the waterworks purify the waste water and deliver the regenerated water to the industries. Obviously the total operation process of water utilization and regeneration for a city or a macroregion is a dynamic, recycling, and repeating system.

In the current paper we examine the performance measurement problem for water utilization and recycling systems, pointing out the fact that a novel method should be proposed to deal with the mentioned problem. To that end, in the remainder of this section we elaborate the subsystems and factors (outputs and inputs) used in the evaluation. In the section to follow, a modified version of the conventional DEA model is developed.

Figure 1 shows a typical operation process of water utilization and recycling for a macroregion. The detailed descriptions for the variables and subsystems are given below.

2.2. Variables. As shown in Figure 1, there are 6 types of principal input and output variables in the water utilization and recycling operation. In fact, since the operation is a very complex one, there are more than 6 types of variables involved in the operation. We will discuss the other notmentioned-here variables later. The six principal input and output variables are given as follows.

2.2.1. Quantity of Fresh Water Used in Industries (QFW or F). Fresh water is supplied by waterworks to the enterprises. It is the initial input of the whole operation. Here we denote the quantity of fresh water by QFW or $F$ for short. Under normal conditions, for a specific water recycling system, with the 
quantity of fresh water increasing, those downstream variables such as the quantities of waste water, emitted waste water, processed water, and regenerated water will also increase.

2.2.2. Quantity of Industrial Waste Water (QIWW or W). Industrial waste water is brought during all types of industrial activities. Obviously, if the waste water cannot be processed and reused, the output variable QIWW or $W$ for short is an undesirable one, and the less quantity of industrial waste water means the better situation, since the industrial waste water is harmful to the environment. However, if the waste water is processed and reused, the situations are totally different. The output variable $W$ becomes a desirable one, since more industrial waste water implies more resources for reuse.

\subsubsection{Quantity of Emitted Waste Water (QEWW or E).} Among the produced industrial waste water by all sorts of enterprises, part of it is emitted directly, while the rest is collected for purification and reuse. Apparently, the emitted part of industrial waste water, denoted by QEWW or E, is absolutely a type of undesirable output. In an ecological economy evaluation study, the best enterprises are those ones which try not to emit or only emit the least waste water directly but still remain to be satisfying enough economic outputs when keeping inputs constant.

2.2.4. Quantity of Processed Waste Water (QPWW or P). As discussed above, part of the industrial waste water is collected for purification and repetitive use. This part, of course, is a useful resource for better utilization of water. In the paper we regard the processed waste water as the unique input in the waste water regeneration subsystem.

\subsubsection{Quantity of Regenerated Waste Water (QRWW or R).}

In the current study, the regenerated waste water plays two roles in our evaluation. Firstly it is an output of waste water regeneration subsystem. Secondly it is transferred from waterworks to industries again for reuse, so it is also an input of fresh water utilization subsystem. If the quantity of regenerated waste water equals to zero, the water circulation and repetitive use will not occur. Therefore we believe QRWW or $R$ is the most special and important variable in the current study. We will discuss the variable in the next section more detailedly.

2.2.6. Quantity of Economic Product (QEP or G). Although there are many outputs in a water utilization and recycling system, such as satisfaction level of urban citizens and employment offered by waterworks, in the current study, we only focus on two types of outputs: one is the quantity of emitted waste water, and the other is the economic product, represented by the total amount of GDP caused by industrial enterprises in a specific region. If we ignore the variable QEP or $G$ for short, we cannot appraise a water utilization and recycling system reasonably and fairly.

It should be announced that there are some other resources involved in the water utilization and recycling system but we had better ignore them. For example, in the fresh water utilization subsystem, several recourses are used to produce economic output and discharge waste water. Those recourses include but are not be limited to energy, capital, labor, time, facilities, and pipelines. We do not take those resources into consideration because of three facts.

Firstly, relative to other resources, water is the most precious scarce one. Some resources are consumed little such as energy, some resources do not vanish such as facilities and pipelines, and some resources are not comparatively expensive or scarce, such as capital, labor, and time. Accordingly, the importance of other resources is inferior to that of fresh water.

Secondly, from the perspective of a macroregion, the relevancy level between fresh water and GDP or waste water is much higher than the relevancy levels between other resources and GDP or waste water. Apparently the quantity of waste water relies on the quantity of fresh water and not the other resources. Moreover, in the water utilization and recycling system, the economic output GDP also mainly depends upon the quantity of fresh water, since other resources in different water recycling systems are often similar or close.

Thirdly, excessive variables cannot bring reasonable results in DEA framework, and the unimportant variables often confuse the appraisement. In addition, the data of other resources usually cannot be easily acquired.

2.3. System Decomposition. Although the water utilization and recycling operation is a dynamic, recycling, and repeating system, we still regard it as a static one temporarily and divide it into three subsystems for better and more convenient analysis. Any two subsystems are related to a specific variable. The three subsystems include the following.

2.3.1. Subsystem A. Fresh water utilization (FWU): this subsystem is operated by the enterprises involved in the water utilization and recycling system. In the fresh water utilization subsystem, enterprises use two types of resources, fresh water and regenerated water, to produce economic output and waste water. As discussed above, although other resources such as energy, capital, and labor are also consumed in the subsystem, we had better ignore them in the current paper.

2.3.2. Subsystem $B$. Waste water emission (WWE): this subsystem is controlled by the urban drainage system. According to the qualities of waste water and the purification abilities of waterworks, the urban drainage system classifies the waste water into two categories, processable and unprocessable. Then the urban drainage system transfers the processable waste water to waterworks for purification and ejects the unprocessable one to some places where useless waste water is collected and disposed. In short, the input of waste water emission subsystem is waste water from subsystem A, and its outputs are processable (processed water, $P$ ) and unprocessable water (emitted waste water, $E$ ).

2.3.3. Subsystem C. Waste water regeneration (WWR): this subsystem is under the charge of waterworks. The processable water is transported to waterworks, and waterworks purify it 
through procedures including sedimentation, filtration, and medication. As a result, the regenerated water is obtained to be used as fresh water.

\section{The Foundational Assumptions}

3.1. The Assumption on Returns to Scales. The current paper is based on the assumption that the three subsystems mentioned in Section 2.3 are constant returns to scales (CRS). In general, there are few production systems which follow strictly constant returns to scales. We insist on the CRS assumption because of two reasons. Firstly, CRS assumption makes the problem much simpler and makes it possible to probe into the inherent rules of operational performance of recycling systems. As will be discussed later, CRS assumption helps find out the relationship of performance of a dynamic system and its similar static system. But contrarily, the variable returns to scales (VRS) assumption will prevent us from capturing an exact estimation to the frontier production ability of a given recycling system. Then it is hard for us to appraise the recycling system reasonably. The second reason why we present the CRS assumption is that, according to experiential observations, the three subsystems are very close to CRS, so the assumption would not mislead the appraisement results.

3.2. The Assumptions on Variable R's Impact on System Operation. As mentioned above, we believe QRWW or $R$ is the most special and important variable in the current study, because it connects subsystem $\mathrm{C}$ with subsystem $\mathrm{A}$ and makes the water utilization and recycling system take on a dynamic one. So, considering the impact from variable $R$ on the whole system is vital to our study.

In subsystem A, there are two types of inputs involved, fresh water $(F)$ and regenerated water $(R)$. Thus compared with variable $F$, we suppose $R$ has the same impact as $F$ on system operation.

In other papers we will discuss the other scenarios about variable $R$ 's impact on system operation, including (1) null impact assumption, (2) full impact assumption, and (3) dull impact assumption. In the current paper, the null impact assumption, full impact assumption, and dull impact assumption are all not suitable to be accepted, so we would not discuss them in the next sections.

\section{Modeling Performance Evaluation for Water Recycling Systems}

Suppose there are $n$ water recycling systems as shown in Figure 1 . We regard them as $n$ decision making units (DMUs). For the $j$ th DMU, the corresponding input and output measures are $F_{j}, G_{j}, W_{j}, E_{j}, P_{j}$, and $R_{j}(j=1, \ldots, n)$, respectively.

In a water recycling system, variable $F$ denotes fresh water and $R$ means regenerated water. Obviously, the equal impact assumption for variable $R$ stands here. Variable $R$ has the same impact as variable $F$ on the subsystem A's operation.

Similar to Figure 3, the dynamic water recycling system is equivalent to a multicirculation production system. Within

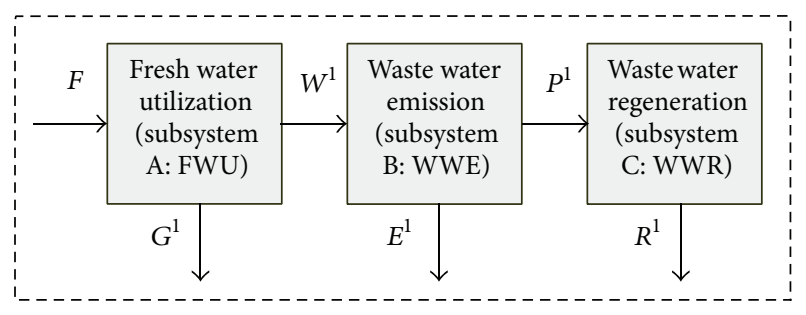

FIgURE 2: The first-circulation equivalent system.

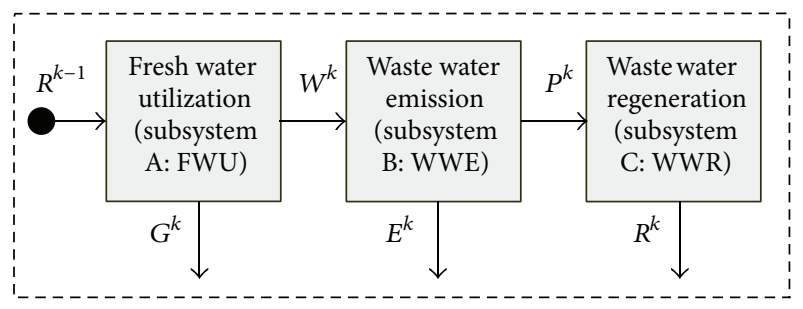

FIgURE 3: The $k$ th-circulation equivalent system.

each circulation there exist three subsystems, different from the equivalent system in Figure 3 where only one subsystem exists in each circulation. The first circulation is seen in Figure 2, and the $k$ th circulation is shown as Figure 3. For the fact that the equivalent system is too complex to be put in a figure, we do not describe it graphically.

In the first-circulation system, as shown in Figure 2, input $F$ is consumed to generate three types of outputs $G^{1}, E^{1}$, and $R^{1}$ through three subsystems.

In the second-circulation system in Figure 3, input $R^{1}$ is used to produce outputs $G^{2}, E^{2}$, and $R^{2}$. Since input $F$ generates $G^{1}, E^{1}$, and $R^{1}$ and the three subsystems are CRS, we conclude that

$$
\begin{gathered}
F: G^{1}=R^{1}: G^{2}, \quad F: E^{1}=R^{1}: E^{2}, \\
F: R^{1}=R^{1}: R^{2} .
\end{gathered}
$$

Let $\xi=R^{1} / F$; then we have $G^{2}=\xi \times G^{1}, E^{2}=\xi \times E^{1}$, and $R^{2}=\xi \times R^{1}$.

Similarly, in the $k$ th-circulation system in Figure 3, input $R^{k-1}$ is used to produce outputs $G^{k}, E^{k}$, and $R^{k}$. Accordingly,

$$
G^{k}=\xi \times G^{k-1}, \quad E^{k}=\xi \times E^{k-1}, \quad R^{k}=\xi \times R^{k-1} .
$$

Thus

$$
\begin{aligned}
& G^{k}=\xi \times G^{k-1}=\xi \times \xi \times G^{k-2}=\cdots=\xi^{k-1} G^{1}, \\
& E^{k}=\xi \times E^{k-1}=\xi \times \xi \times E^{k-2}=\cdots=\xi^{k-1} E^{1}, \\
& R^{k}=\xi \times R^{k-1}=\xi \times \xi \times R^{k-2}=\cdots=\xi^{k-1} R^{1} .
\end{aligned}
$$

Let input-output pairs $\mathrm{APA}_{2 d}$ and $\mathrm{FPA}_{2 d}$ be the actual production ability and frontier production ability, and let $\mathrm{AO}_{2 d}$ and $\mathrm{FO}_{2 d}$ be the actual output and frontier output while keeping inputs unchanged. 
Therefore $A O_{2 d}$ equals the sum of outputs in all of circulations. Then

$$
\begin{aligned}
A O_{2 d} & =\left(A G_{2 d}, A E_{2 d}, A R_{2 d}\right), \\
A G_{2 d} & =\lim _{t \rightarrow \infty}\left(G^{1}+G^{2}+\cdots+G^{t}\right) \\
& =\lim _{t \rightarrow \infty}\left(G^{1}+\xi G^{1}+\cdots+\xi^{t-1} G^{1}\right) \\
& =\lim _{t \rightarrow \infty}\left(1+\xi+\xi^{2}+\cdots+\xi^{t-1}\right) G^{1} \\
& =\lim _{t \rightarrow \infty} \frac{1-\xi^{t}}{1-\xi} G^{1}, \\
A E_{2 d} & =\lim _{t \rightarrow \infty} \frac{1-\xi^{t}}{1-\xi} E^{1}, \\
A R_{2 d} & =\lim _{t \rightarrow \infty} \frac{1-\xi^{t}}{1-\xi} R^{1} .
\end{aligned}
$$

If $\xi<1, A G_{2 d}=(1 /(1-\xi)) G^{1}=\left(1 /\left(\left(1-R^{1}\right) / F\right)\right) G^{1}=(F /(F-$ $\left.\left.R^{1}\right)\right) G^{1}, A E_{2 d}=\left(F /\left(F-R^{1}\right)\right) E^{1}$, and $A R_{2 d}=\left(F /\left(F-R^{1}\right)\right) R^{1}$. Otherwise, if $\xi \geq 1, A G_{2 d} \rightarrow \infty, A E_{2 d} \rightarrow \infty$, and $A R_{2 d} \rightarrow$ $\infty$.

Next we consider the frontier output $F O_{2 d}$.

In the first-circulation system, as shown in Figure 2, under the existing optimal technique constraints, input $F$ generates the maximum outputs as $\widehat{G}^{1}=G^{1} / \theta_{A}^{\mathrm{CCR}}, \widehat{E}^{1}=E^{1}$ / $\left(\theta_{A}^{\mathrm{CCR}} \theta_{B}^{\mathrm{CCR}}\right)$, and $\widehat{R}^{1}=R^{1} /\left(\theta_{A}^{\mathrm{CCR}} \theta_{B}^{\mathrm{CCR}} \theta_{C}^{\mathrm{CCR}}\right)$.

In the second-circulation system, the maximum input is $\widehat{R}^{1}$, not $R^{1}$. Since input $F$ generates the maximum outputs as $\widehat{G}^{1}=G^{1} / \theta_{A}^{\mathrm{CCR}}, \widehat{E}^{1}=E^{1} /\left(\theta_{A}^{\mathrm{CCR}} \theta_{B}^{\mathrm{CCR}}\right)$, and $\widehat{R}^{1}=R^{1} /$ $\left(\theta_{A}^{\mathrm{CCR}} \theta_{B}^{\mathrm{CCR}} \theta_{C}^{\mathrm{CCR}}\right)$ and the subsystems are CRS, we conclude that

$$
F: \widehat{G}^{1}=\widehat{R}^{1}: \widehat{G}^{2}, \quad F: \widehat{E}^{1}=\widehat{R}^{1}: \widehat{E}^{2}, \quad F: \widehat{R}^{1}=\widehat{R}^{1}: \widehat{R}^{2} .
$$

Let $\zeta=\widehat{R}^{1} / F=R^{1} /\left(F \theta_{A}^{\mathrm{CCR}} \theta_{B}^{\mathrm{CCR}} \theta_{C}^{\mathrm{CCR}}\right)$; then we have

$$
\widehat{G}^{2}=\zeta \times \widehat{G}^{1}, \quad \widehat{E}^{2}=\zeta \times \widehat{E}^{1}, \quad \widehat{R}^{2}=\zeta \times \widehat{R}^{1} .
$$

Similarly, in the $k$ th-circulation system, we have

$$
\widehat{G}^{k}=\zeta \times \widehat{G}^{k-1}, \quad \widehat{E}^{k}=\zeta \times \widehat{E}^{k-1}, \quad \widehat{R}^{k}=\zeta \times \widehat{R}^{k-1} .
$$

Thus

$$
\begin{gathered}
\widehat{G}^{k}=\zeta \times \widehat{G}^{k-1}=\zeta \times \zeta \times \widehat{G}^{k-2}=\cdots=\zeta^{k-1} \widehat{G}^{1}, \\
\widehat{E}^{k}=\zeta \times \widehat{E}^{k-1}=\zeta \times \zeta \times \widehat{E}^{k-2}=\cdots=\zeta^{k-1} \widehat{E}^{1}, \\
\widehat{R}^{k}=\zeta \times \widehat{R}^{k-1}=\zeta \times \zeta \times \widehat{R}^{k-2}=\cdots=\zeta^{k-1} \widehat{R}^{1} .
\end{gathered}
$$

Therefore $\mathrm{FO}_{4 d}$ equals the sum of frontier outputs in all of circulations. Then

$$
\begin{aligned}
F O_{2 d} & =\left(F G_{2 d}, F E_{2 d}, F R_{2 d}\right), \\
F G_{2 d} & =\lim _{t \rightarrow \infty}\left(\widehat{G}^{1}+\widehat{G}^{2}+\cdots+\widehat{G}^{t}\right) \\
& =\lim _{t \rightarrow \infty}\left(\widehat{G}^{1}+\zeta \widehat{G}^{1}+\cdots+\zeta^{t-1} \widehat{G}^{1}\right) \\
& =\lim _{t \rightarrow \infty}\left(1+\zeta+\zeta^{2}+\cdots+\zeta^{t-1}\right) \widehat{G}^{1} \\
& =\lim _{t \rightarrow \infty} \frac{1-\zeta}{1-\zeta} \widehat{G}^{1}, \\
F E_{2 d} & =\lim _{t \rightarrow \infty} \frac{1-\zeta}{1-\zeta} \widehat{E}^{1}, \\
F R_{2 d} & =\lim _{t \rightarrow \infty} \frac{1-\zeta}{1-\zeta} \widehat{R}^{1} .
\end{aligned}
$$

If $\zeta<1$

$$
\begin{aligned}
F G_{2 d}= & \frac{1}{1-\zeta} \widehat{G}^{1}=\frac{1}{1-R^{1} /\left(F \theta_{A}^{\mathrm{CCR}} \theta_{B}^{\mathrm{CCR}} \theta_{C}^{\mathrm{CCR}}\right)} \times \frac{G^{1}}{\theta_{A}^{\mathrm{CCR}}} \\
= & \frac{F \theta_{A}^{\mathrm{CCR}} \theta_{B}^{\mathrm{CCR}} \theta_{C}^{\mathrm{CCR}}}{F \theta_{A}^{\mathrm{CCR}} \theta_{B}^{\mathrm{CC}} \theta_{C}^{\mathrm{CR}}-R^{1}} \times \frac{G^{1}}{\theta_{A}^{\mathrm{CCR}}} \\
= & \frac{F G^{1} \theta_{B}^{\mathrm{CCR}} \theta_{C}^{\mathrm{CCR}}}{F \theta_{A}^{\mathrm{CCR}} \theta_{B}^{\mathrm{CCR}} \theta_{C}^{\mathrm{CCR}}-R^{1}}, \\
F E_{2 d}= & \frac{1}{1-\zeta} \widehat{E}^{1}=\frac{1}{1-R^{1} /\left(F \theta_{A}^{\mathrm{CCR}} \theta_{B}^{\mathrm{CCR}} \theta_{C}^{\mathrm{CCR}}\right)} \times \frac{E^{1}}{\theta_{A}^{\mathrm{CCR}} \theta_{B}^{\mathrm{CCR}}} \\
= & \frac{F \theta_{A}^{\mathrm{CCR}} \theta_{B}^{\mathrm{CCR}} \theta_{C}^{\mathrm{CCR}}}{F \theta_{A}^{\mathrm{CCR}} \theta_{B}^{\mathrm{CCR}} \theta_{C}^{\mathrm{CCR}}-R^{1}} \times \frac{E^{1}}{\theta_{A}^{\mathrm{CCR}} \theta_{B}^{\mathrm{CCR}}} \\
= & \frac{F E^{1} \theta_{C}^{\mathrm{CCR}}}{F \theta_{A}^{\mathrm{CCR}} \theta_{B}^{\mathrm{CCR}} \theta_{C}^{\mathrm{CCR}}-R^{1}}, \\
F R_{2 d}= & \frac{1}{1-\zeta} \widehat{R}^{1}=\frac{1}{1-R^{1} /\left(F \theta_{A}^{\mathrm{CCR}} \theta_{B}^{\mathrm{CCR}} \theta_{C}^{\mathrm{CCR}}\right)} \\
& \times \frac{R^{1}}{\theta_{A}^{\mathrm{CCR}} \theta_{B}^{\mathrm{CCR}} \theta_{C}^{\mathrm{CCR}}} \\
= & \frac{F \theta_{A}^{\mathrm{CCR}} \theta_{B}^{\mathrm{CCR}} \theta_{C}^{\mathrm{CCR}}}{F \theta_{A}^{\mathrm{CCR}} \theta_{B}^{\mathrm{CC}} \theta_{C}^{\mathrm{CR}}-R^{1}} \times \frac{R_{A}}{\theta_{A}^{\mathrm{CCR}} \theta_{B}^{\mathrm{CCR}} \theta_{C}^{\mathrm{CCR}}} \\
= & \frac{F \theta_{A}^{\mathrm{CCR}} \theta_{B}^{\mathrm{CCR}} \theta_{C}^{\mathrm{CCR}}-R^{1}}{}
\end{aligned}
$$

Otherwise, if $\zeta \geq 1, F G_{2 d} \rightarrow \infty, F E_{2 d} \rightarrow \infty$, and $F R_{2 d} \rightarrow$ $\infty$.

4.1. Performance Evaluation Model. The above analysis has let us know all system's actual production ability and frontier 
production ability, although we only acquire the data from the first-circulation system.

Thus we appraise a recycling system's efficiency by the following programming:

$$
\begin{aligned}
& \beta_{d}^{\mathrm{CPS}}= \max \frac{U^{T}\left(A G_{4 d}, A E_{4 d}\right)}{V^{T} F_{d}} \\
& \text { s.t. } \frac{U^{T}\left(A G_{4 j}, A E_{4 j}\right)}{V^{T} F_{j}} \leq 1, \\
& \frac{U^{T}\left(F G_{4 j}, F E_{4 j}\right)}{V^{T} F_{j}} \leq 1, \\
& U, V \geq 0 .
\end{aligned}
$$

Remark 1. It should be announced that we drop output variable $R\left(A R_{3 d}, F R_{3 d}, A R_{4 d}\right.$, and $\left.F R_{4 d}\right)$ in the performance evaluation models $(A)$, since this type of output is not really an output of the system, and it will disappear during the infinite-circulation repetition.

Remark 2. In the current paper, output $E$ is an undesirable output, so we should transform it into a desirable one before computation.

The DEA research on dealing with undesirable outputs is mainly divided into three groups: including the reciprocal measure approach by Färe et al. [12], regarding undesirable outputs as inputs by Reinhard et al. [17], and turning undesirable outputs to be positive desirable outputs by a linear monotone decreasing transformation by Seiford and Zhu [18]. We employ the first technique in the current approach.

With regard to the optimal solution to (A), $\beta_{d}^{\text {CPS }}$, we have the following conclusions.

Conclusion 1. $\zeta \geq \xi$ because $\zeta=\xi /\left(\theta_{A}^{\mathrm{CCR}} \theta_{B}^{\mathrm{CCR}} \theta_{C}^{\mathrm{CCR}}\right)$ and $\theta_{A}^{\mathrm{CCR}} \theta_{B}^{\mathrm{CCR}} \theta_{C}^{\mathrm{CCR}} \leq 1$.

Conclusion 2. If $\xi<1$ and $\zeta \geq 1$, the actual outputs are finite, and frontier outputs are infinite, $\beta_{d}^{\mathrm{CPS}} \rightarrow 0$.

Conclusion 3. If $\xi<1$ and $\zeta<1,0<\beta_{d}^{\mathrm{CPS}} \leq 1$.

Conclusion 4. If $\xi \geq 1$ and $\zeta \geq 1$, it is hard to compute programming (A).

\section{Results and Discussions}

In the current paper we focus on Chinese provincial water recycling and utilization systems and try to give a reasonable and fair appraisement to the operational performance of those provincial systems. There are 31 provinces in Chinese mainland. Here 29 provincial water recycling and utilization systems are included in the evaluation except Guizhou and Xizang, because no data are available about these two provinces. There are six variables involved in the computation as shown in Section 2.2. All the data are from National Bureau of Statistics of China (http://www.stats.gov.cn/tjsj/). Table 1 reports the computation results.

5.1. Result Analysis in 2003. The efficiency scores for all Chinese provincial water recycling and utilization systems in 2003 are shown in Table 1 . The table contains the following information:

columns 1 and 2: the number and name of each provincial water recycling and utilization system;

columns 3-5: the CCR efficiency scores for each subsystem;

columns 6 and 7: the multipliers $\xi$ and $\zeta$ in the infinite circulations while considering actual and frontier final outputs, respectively;

column 8: the DEA efficiency scores of the overall system from model (A).

We find out some facts from the results in Table 1, as follows.

(1) In 2003, for each province, the multiplier $\xi$ is very small, obviously less than the multiplier $\zeta$. The values for $\xi$ for all provinces stand in the interval [0.00005, 0.058]. Chongqing (number 22) is the smallest, and Heilongjiang (number 8) is the largest. Chongqing (number 22) has a multiplier 0.00005 which means that the regenerated water only equals 0.00005 times of fresh water in each circulation of water utilization and recycling. Although Heilongjiang (number 8 ) has the highest multiplier; only $5.8 \%$ of water is reused. The fact shows that the water utilization efficiency is still low in every province in China.

(2) In 2003, for each province, the multiplier $\zeta$ ranges from 0.0009 (Qinghai, number 27) to 0.2944 (Hubei, number 17), with the majority of provinces between 0.25 and 0.3 , much higher than the multiplier $\xi$. In the current study, the multiplier $\zeta$ reflects the possible maximum percentage of fresh water which would be reused in each circulation of water utilization and recycling if all subsystems are improved efficiently under the existing technique constraints. Therefore, under the existing technique constraints, the optimal reuse ratio is $29.44 \%$ in today's China. The " $>30 \%$ " reuse ratio in China would not come true, except when the existing technique is improved greatly.

(3) The DEA efficiency score of the overall system from model (A) in the rightmost column is less than the CCR efficiency for subsystem A in column 3, which is also shown in Figure 4. The reason lies on the fact that the overall efficiency in the rightmost column considers the reuse of resources. The output $G$ is produced in subsystem A, so the frontier output for $G$ is determined by $G$ and the CCR efficiency of subsystem $A$ if no reuse happens. Obviously, considering the reuse scenario, the frontier output for $G$ will become much larger, so the overall efficiency should be less than subsystem A's CCR efficiency. 
TABLE 1: Results in 2003.

\begin{tabular}{|c|c|c|c|c|c|c|c|}
\hline Number & Province & $\theta_{A}^{\mathrm{CCR}}$ & $\theta_{B}^{\mathrm{CCR}}$ & $\theta_{\mathrm{C}}^{\mathrm{CCR}}$ & $\xi$ & $\zeta$ & $\beta_{d}^{\mathrm{CPS}}$ \\
\hline 01 & Beijing & 0.1666 & 0.6059 & 0.2099 & 0.006 & 0.2808 & 0.0939 \\
\hline 02 & Tianjin & 0.9747 & 0.5461 & 0.0306 & 0.0043 & 0.2609 & 0.7072 \\
\hline 03 & Hebei & 0.391 & 0.5351 & 0.1203 & 0.0064 & 0.2531 & 0.287 \\
\hline 04 & Shanxi & 0.1427 & 0.5906 & 0.0421 & 0.0009 & 0.2452 & 0.1073 \\
\hline 05 & Inner Mongolia & 0.1953 & 0.5276 & 0.0102 & 0.0003 & 0.2379 & 0.1445 \\
\hline 06 & Liaoning & 0.3055 & 0.473 & 0.1178 & 0.005 & 0.2926 & 0.1348 \\
\hline 07 & Jilin & 0.1428 & 0.4236 & 0.0113 & 0.0002 & 0.278 & 0.073 \\
\hline 08 & Heilongjiang & 0.3233 & 0.6225 & 1 & 0.058 & 0.2883 & 0.1642 \\
\hline 09 & Shanghai & 0.4286 & 1 & 0.1526 & 0.0183 & 0.2796 & 0.2509 \\
\hline 10 & Jiangsu & 0.3953 & 0.8427 & 0.4389 & 0.0405 & 0.2769 & 0.2457 \\
\hline 11 & Zhejiang & 1 & 0.5546 & 0.004 & 0.0006 & 0.2524 & 0.748 \\
\hline 12 & Anhui & 0.609 & 0.5726 & 0.0215 & 0.0022 & 0.2894 & 0.287 \\
\hline 13 & Fujian & 0.6424 & 0.4832 & 0.0144 & 0.0011 & 0.2506 & 0.4764 \\
\hline 14 & Jiangxi & 1 & 0.2586 & 0.0588 & 0.0044 & 0.2907 & 0.4212 \\
\hline 15 & Shandong & 0.3158 & 0.5996 & 0.0952 & 0.0036 & 0.1992 & 0.2415 \\
\hline 16 & Henan & 0.2687 & 0.4209 & 0.0094 & 0.0003 & 0.2728 & 0.1735 \\
\hline 17 & Hubei & 0.6733 & 0.4444 & 0.2453 & 0.0216 & 0.2944 & 0.259 \\
\hline 18 & Hunan & 0.3978 & 0.3247 & 0.6547 & 0.0246 & 0.2914 & 0.1798 \\
\hline 19 & Guangdong & 0.4945 & 0.3306 & 0.1064 & 0.005 & 0.2886 & 0.2396 \\
\hline 20 & Guangxi & 0.4111 & 0.4008 & 0.0374 & 0.0018 & 0.2921 & 0.1275 \\
\hline 21 & Hainan & 0.1765 & 1 & 0.0538 & 0.0019 & 0.198 & 0.1418 \\
\hline 22 & Chongqing & 1 & 0.3272 & 0.0006 & $5 E-05$ & 0.2547 & 0.586 \\
\hline 23 & Sichuan & 0.6391 & 0.2987 & 0.5923 & 0.0331 & 0.2929 & 0.0965 \\
\hline 24 & Yunnan & 0.7439 & 0.7621 & 0.1234 & 0.0196 & 0.2802 & 0.0772 \\
\hline 25 & Shaanxi & 0.7382 & 0.2814 & 0.2658 & 0.0156 & 0.2829 & 0.2671 \\
\hline 26 & Gansu & 0.5303 & 0.4386 & 0.0781 & 0.005 & 0.2764 & 0.099 \\
\hline 27 & Qinghai & 0.5101 & 0.8175 & 0.6216 & 0.0002 & 0.0009 & 0.3979 \\
\hline 28 & Ningxia & 0.3932 & 0.5431 & 0.0043 & 0.0001 & 0.1307 & 0.3281 \\
\hline 29 & Xinjiang & 1 & 0.7221 & 0.4115 & 0.0435 & 0.1464 & 0.8268 \\
\hline
\end{tabular}

(4) The DEA efficiency score of the overall system from model (A) in the rightmost column is less than the product of the CCR efficiencies for subsystems A and $\mathrm{B}$ in columns 3 and 4 . The fact is apparent since the overall efficiency is less the A's efficiency and another fact that B's efficiency is less than 1 . We mention this because the frontier target for another output $E$ is determined by itself and the CCR efficiencies of subsystems A and B. The reason for the fact is similar to above.

(5) The overall efficiency scores lie in $[0.073,0.8268]$ corresponding to Jilin (number 7 ) and Xinjiang (number 29), as shown in the rightmost column. Jilin has the least overall efficiency of 0.073 , which means its outputs can be enhanced to about 14 times of themselves during the whole life circle of the water utilization and recycling. Jilin's CCR efficiency for subsystem $\mathrm{A}$ is 0.1428 , implying that the output $G$ can be enhanced to about 7 times of itself if no reuse happens. Therefore reuse of resources can improve the potential of outputs extremely greatly.

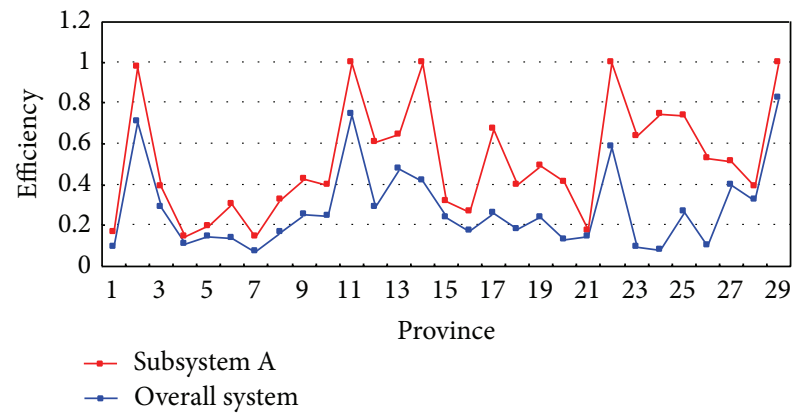

Figure 4: Efficiency analysis in 2003.

5.2. Results Comparison during 2003-2006. We also obtain the results during 2003-2006 for Chinese provincial water utilization and recycling systems. Some interesting conclusions are drawn in the following paragraphs.

(1) The multipliers $\zeta$ and $\xi$ have not be enhanced greatly during the past several years. In 2003, $\zeta$ ranges in 


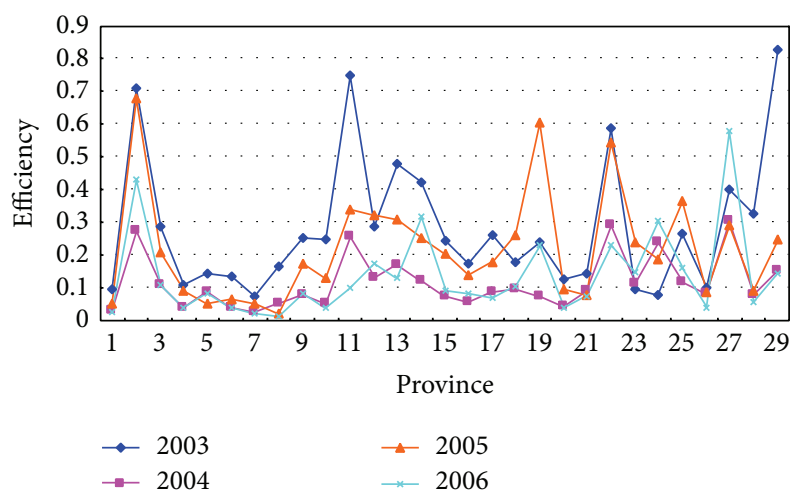

FIGURE 5: Overall efficiency comparison during 2003-2006.

$[0.00005,0.058]$ and $\xi$ in $[0.0009,0.2944]$; in $2004, \zeta$ ranges in $[0.00006,0.0403]$ and $\xi$ in $[0.1188,0.486]$; in $2005, \zeta$ ranges in $[0.00007,0.0452]$ and $\xi$ in $[0.2284$, $0.3894]$; in $2006, \zeta$ ranges in $[0.00006,0.028]$ and $\xi$ in $[0.2914,0.6933]$. Apparently, the relatively stable values for multipliers $\zeta$ and $\xi$ mean that the reuse ratio for water does not have distinct improvement.

(2) Figure 5 describes the overall efficiency scores for all Chinese provinces during 2003-2006. It is found out that the efficiency scores are very stable from 2003 to 2006 for almost all provinces. There are some provinces which always have high efficiency scores such as Tianjin (number 2), Zhejiang (number 11), Chongqing (number 22), Qinghai (number 27), and Xinjiang (number 29), while some have always low scores such as Beijing (number 1), Liaoning (number 6), Jilin (number 7), Heilongjiang (number 8), Guangxi (number 20), and Gansu (number 26). Those provinces with higher efficiency scores should be encouraged because the higher efficiency scores usually imply the higher reuse ratio of resources, while the opposite provinces should be criticized.

\section{Conclusions}

The current paper examines the operational performances of Chinese provincial water utilization and recycling system during 2003-2006. A water utilization and recycling system is a typical infinitely repeated and recycling system, and the existing approaches cannot measure the performance of this classification of systems effectively. Thus the current paper advances an "equivalent multicirculation system" approach to estimate the actual and frontier output of those systems while keeping the input unchanged. In fact, the proposed approach is also to seek the actual and frontier production abilities during the total life circle of recycling systems. Once the actual production abilities and frontier production abilities are obtained, the technical efficiencies for the systems can be computed easily through traditional models.

As a matter of fact, the current paper is a primary and exploratory study on the subject. It has been emphasized that the proposed method is based on two assumptions: one is that each subsystem is CRS and the other is that the linkage variables (connecting the first and last subsystems) have the same impact on the production of the first subsystem as the initial inputs. In the case study of the current paper, the two assumptions approximately stand true. So we think that the results in the two tables are believable. However, in other real world scenarios, the two assumptions do not always stand true. That is to say, further researches on the topic are in urgently needed.

The results of Chinese provincial water utilization and recycling system are also worthy of notice. China is a developing industrial power and is famous as a world factory. The development of industry results in a great deal of resources being consumed. The increasing resource demand leads to high price of materials and energy, as well as destroying the environment. To avoid the potential economic recession and ecological disaster, China government implements National Sustainable Development Strategy to save resources to accelerate economy. Water is a scarce resource in China. The current study shows that the reuse level of water in China is still very low. Larger quantity and higher capacity of water works are needed in every province in China.

\section{Conflict of Interests}

The authors declare that there is no conflict of interests regarding the publication of this paper.

\section{Acknowledgments}

The authors would like to thank National Natural Science Foundation of China (Grant nos. 71322101, 71271195, 71110107024, and 71121061) and USTC Fund for Innovation Teams (WK2040160008) for their financial support.

\section{References}

[1] Z. R. Zhong, "Natural resources planning, management, and sustainable use in China," Resources Policy, vol. 25, no. 4, pp. 211-220, 1999.

[2] O. Varis and P. Vakkilainen, "China's 8 challenges to water resources management in the first quarter of the 21st century," Geomorphology, vol. 41, no. 2, pp. 93-104, 2001.

[3] E. Thanassoulis, "Use of data envelopment analysis in the regulation of UK water utilities: water distribution," European Journal of Operational Research, vol. 126, no. 2, pp. 436-453, 2000.

[4] A. Charnes, W. W. Cooper, and E. Rhodes, "Measuring the efficiency of decision making units," European Journal of Operational Research, vol. 2, no. 6, pp. 429-444, 1978.

[5] L. Jin and W. Young, "Water use in agriculture in china: importance, challenges, and implications for policy," Water Policy, vol. 3, no. 3, pp. 215-228, 2001.

[6] X. Mo, S. Liu, Z. Lin, Y. Xu, Y. Xiang, and T. R. McVicar, "Prediction of crop yield, water consumption and water use efficiency with a SVAT-crop growth model using remotely sensed data on the North China Plain," Ecological Modelling, vol. 183, no. 2-3, pp. 301-322, 2005.

[7] Y. Huang, L. Chen, B. Fu, Z. Huang, and J. Gong, "The wheat yields and water-use efficiency in the Loess Plateau: straw mulch 
and irrigation effects," Agricultural Water Management, vol. 72, no. 3, pp. 209-222, 2005.

[8] J.-L. Hu, S.-C. Wang, and F.-Y. Yeh, "Total-factor water efficiency of regions in China," Resources Policy, vol. 31, no. 4, pp. 217-230, 2006.

[9] F. Nasiri and G. Huang, "A fuzzy decision aid model for environmental performance assessment in waste recycling," Environmental Modelling and Software, vol. 23, no. 6, pp. 677-689, 2008.

[10] R. Färe and S. Grosskopf, "Network DEA," Socio-Economic Planning Sciences, vol. 34, no. 1, pp. 35-49, 2000.

[11] H. F. Lewis and T. R. Sexton, "Network DEA: efficiency analysis of organizations with complex internal structure," Computers and Operations Research, vol. 31, no. 9, pp. 1365-1410, 2004.

[12] R. Färe, S. Grosskopf, C. A. K. Lovell, and C. Pasurka, "Multilateral productivity comparisons when some outputs are undesirable: a nonparametric approach," The Review of Economics and Statistics, vol. 71, pp. 90-98, 1989.

[13] R. Färe, S. Grosskopf, C. A. K. Lovell, and S. Yaisawarng, "Derivation of shadow prices for undesirable outputs: a distance function approach," The Review of Economics and Statistics, vol. 75, pp. 374-380, 1993.

[14] A. Amirteimoori, S. Kordrostami, and M. Sarparast, "Modeling undesirable factors in data envelopment analysis," Applied Mathematics and Computation, vol. 180, no. 2, pp. 444-452, 2006.

[15] Z. Hua, Y. Bian, and L. Liang, "Eco-efficiency analysis of paper mills along the Huai River: an extended DEA approach," Omega, vol. 35, no. 5, pp. 578-587, 2007.

[16] L. Liang, Y. Li, and S. Li, "Increasing the discriminatory power of DEA in the presence of the undesirable outputs and large dimensionality of data sets with PCA," Expert Systems with Applications, vol. 36, no. 3, part 2, pp. 5895-5899, 2009.

[17] S. Reinhard, C. A. K. Lovell, and G. J. Thijssen, "Environmental efficiency with multiple environmentally detrimental variables; estimated with SFA and DEA," European Journal of Operational Research, vol. 121, no. 2, pp. 287-303, 2000.

[18] L. M. Seiford and J. Zhu, "Modeling undesirable factors in efficiency evaluation," European Journal of Operational Research, vol. 142, no. 1, pp. 16-20, 2002. 


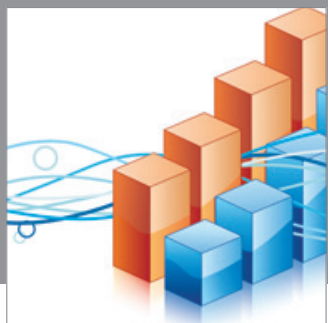

Advances in

Operations Research

mansans

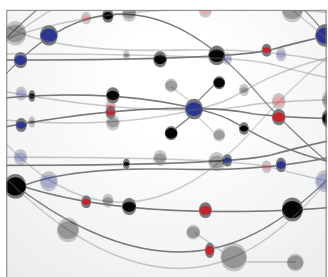

The Scientific World Journal
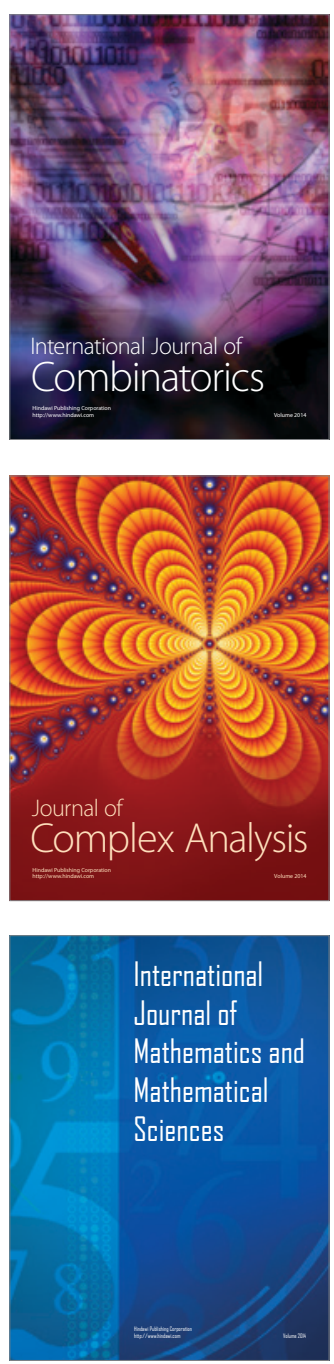
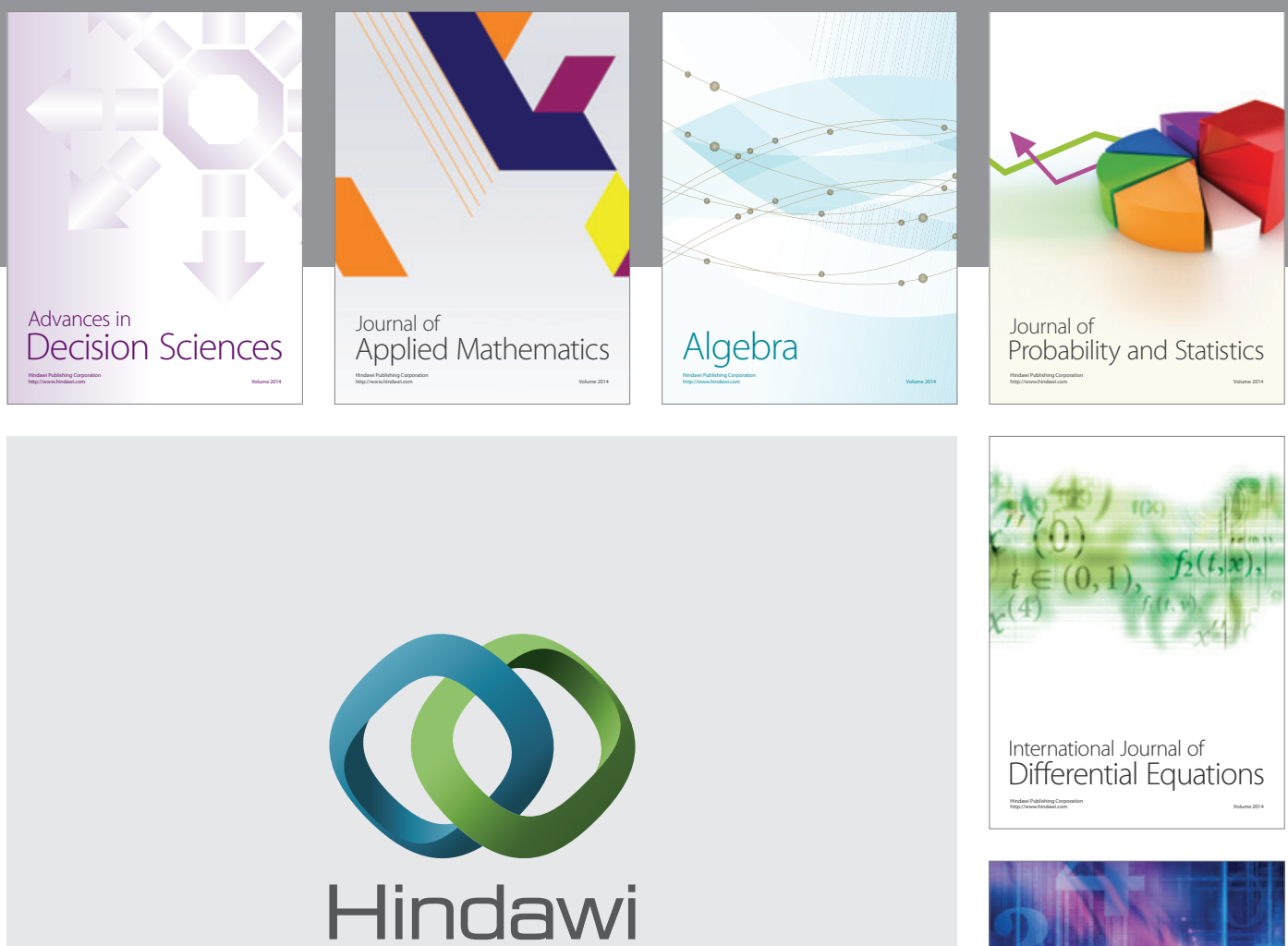

Submit your manuscripts at http://www.hindawi.com
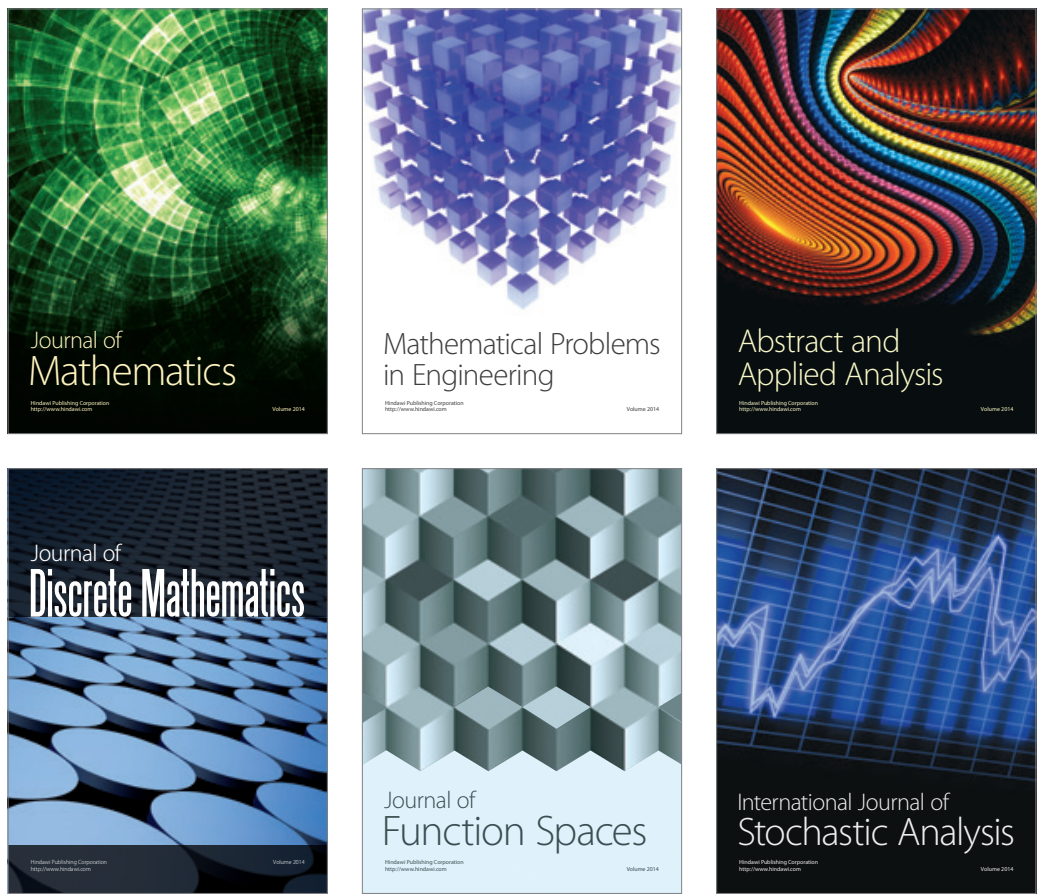

Journal of

Function Spaces

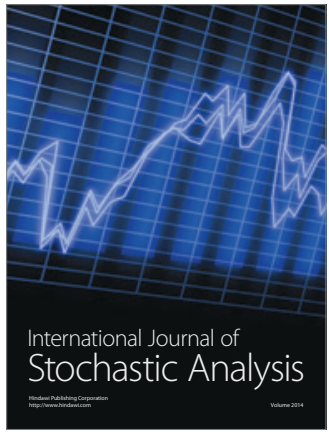

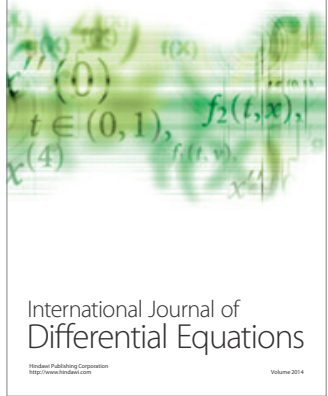
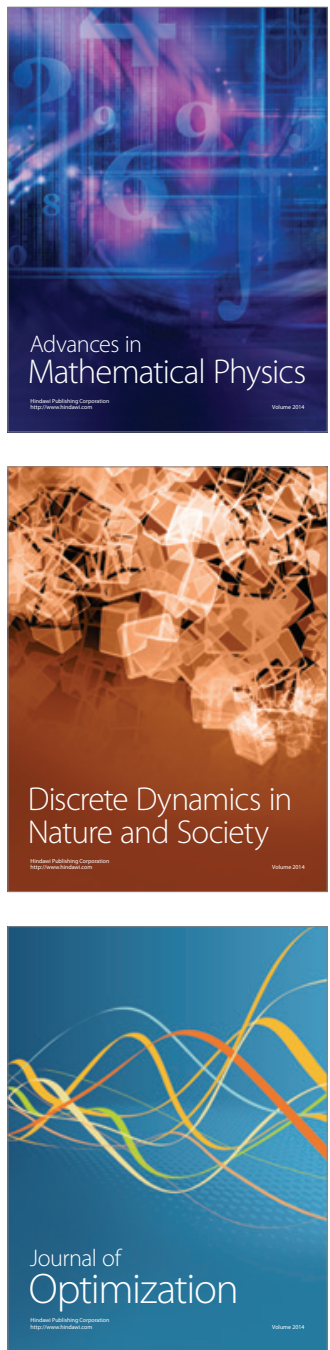